\title{
PS-558
}

\section{IDENTIFICATION OF ORGANIZATIONAL METAPHORS IN BRAZILIAN COMPANIES USING FUZZY CLUSTERING}

Angel Cobo Ortega (Universidad de Cantabria, Spain) - acobo@unican.es

Rocío Rocha (Universidad de Cantabria, Spain) - eliana.rocha@unican.es

Adolfo Alberto Vanti (Universidade do Vale do Rio dos Sinos) - avanti@unisinos.br

Gustavo Schneider (Universidade do Vale do Rio dos Sinos) - gus.schneider@hotmail.com

Most organization and management theories are based on implicit images or metaphors. The aim of this work was to identify the presence of images on a sample of 61 Brazilian companies located in the state of Rio Grande do Sul. For this purpose the results of a questionnaire answered by 198 employees of companies in the sample were analyzed using fuzzy data mining techniques. The results shown that it is difficult to find a clear image in most organizations. In most cases characteristics of different images or metaphors are observed, so soft computing techniques are particularly appropriate for this type of analysis.

Keywords: organizational images, soft clustering, data mining. 


\section{Introduction}

Images are metaphors that allow an interpretation of what is happening with organizational culture. As Morgan (1986) stated, there are 8 images, but in this paper they were reduced to 7 images due to the fact that metaphor Culture already covers Culture and Brains metaphors.

The study of organizational images is widespread on management courses because they provide a very detailed view from organizational studies. However, when it is narrowed to a sector or regional study this kind of approach becomes limited. To overcome the limitation this study presents an approach using Data Mining and Soft Clustering techniques to understand what can happen on organizational culture environment through images in a large number of companies.

The developed application reached 61 enterprises. But this method can be extended to a significant number of companies, coming to a complete study for a country. The sections it this paper are theoretical basis, methodological aspects, case study and conclusions.

\section{Images of Organizations}

All theories of organization and management are based on implicit images or metaphors that persuade us to see, understand, and imagine situations in partial ways (MORGAN, 1986). This author believes that one can better understand organizations by recognizing metaphors that prompt one to view organization through a particular lens. Metaphors play a paradoxical role: they are vital to understanding and highlighting certain aspects of organizations, while at the same time they restrict understanding by back grounding or ignoring others. Morgan illustrates his ideas by exploring eight archetypical metaphors of organization: Machines, Organisms, Brains, Cultures, Political Systems, Psychic Prisons, Flux and Transformation, Instruments of Domination. In this work, brains and culture metaphors are considered as a single image, so 7 metaphors or images are analyzed.

Mechanistic (M): Organizations that impose rigid routines and patterns, hierarchically distributed. Dealings are impersonal and control of the organization is bureaucratic. Because it is very predictable, it is no longer regarded as ideal, even in stable and authoritarian institutions. This style also presents difficulties for innovation.

Psychic Prisons (PP): Inflexibility is a characteristic of this image, becoming a prisoner of past events, allied to fundamental attitudes by their idealizers. Some of their traps are false assumptions, rules without questioning and fanaticism around the charisma of the leader.

Political Systems (PS): This view is not often in the interest of the group and often favors authoritarian executives. This includes companies with participatory management that is encompassed in political systems because although there is a certain distribution of power, the central objective will be executed by both subordinates and the owners of the capital.

Instruments of Domination (ID): In organizations viewed as instruments of domination, the employees and managers completely dedicate themselves to the company. They feel insecure about their employment and experience a lot stress on the job. 
Organisms (0): The fundamental principal of organisms is that it is based on the employees' intellectual capital. Motivation is a substantial factor. Because of constant innovation and deadlines, the employees tend to obey a biological clock because there are targets to reach and constantly innovations to develop.

Brain/Cibernetic (C): Intellectual capital is highly valued and is constantly being stimulated to improve. Decision-making needs to be done "through formal or temporary processes, producing policies and plans that offer a point of reference or a structure for information processing" (JOHANN, 2008, p.33). The definition of cybernetic is given due to the fact that information technology is permanently present, which ensures better conditions in the review of political norms and procedures, addition to learning how to absorb changes in the environment.

Flux and Transformation (FT): Organizations that best mirror flux and transformation are those that modify and evolve to conform to change and evolution in the environment. Their survival depends on their internal and external environments

These images represent the employees behavior as Morgan (1986) stated. Analysis can be performed properly with this approach considering organizational case studies but has some limitations for sector and regional studies using the same criteria. To balance it this paper proposes application of Data Mining (DM) and Soft Clustering techniques that are presented below.

\section{Data mining and soft clustering}

Simply stated, data mining refers to extracting or "mining" knowledge from large amounts of data (HAN; KAMBER, 2006). This area has attracted a great deal of attention in the information industry and in society as a whole in recent years and Data Mining techniques has been applied in a wide variety of areas.

Cluster analysis or clustering is a main task of explorative data mining, and a common technique for statistical data analysis used in many field. Data clustering is the process of dividing data elements into classes or clusters so that items in the same class are as similar as possible, and items in different classes are as dissimilar as possible (WITTEN; FRANK, 2005).

Several approaches exist to clustering. A widespread accepted classification scheme subdivides these techniques in two main groups: hard (crisp) or soft (fuzzy) clustering. In hard clustering, data is divided into distinct clusters, where each data element belongs to exactly one cluster, however in fuzzy clustering, data elements can belong to more than one cluster, and associated with each element is a set of membership levels that indicate the strength of the association between that data element and a particular cluster. Due to the fuzzy nature of many practical problems, a number of fuzzy clustering methods have been developed following the general fuzzy set theory strategies outlined by Zadeh (1965). Fuzzy set theory deals with the representation of classes whose boundaries are not well defined. The key idea is to associate a membership function that takes values in the interval $[0,1]$, with 0 corresponding to no membership in the class a 1 corresponding to full membership. Thus, membership is a notion intrinsically gradual instead of abrupt as in conventional Boolean logic. 
The most known method of fuzzy clustering is the Fuzzy c-Means method (FCM), initially proposed by Dunn (1973) and generalized by Bezdek (1981) and other authors; in Kruse et al. (1999) an overview is presented. The FCM is based on an optimization problem which objective function is defined as:

where $\left\{x_{1}, x_{2}, \ldots, x_{n}\right\}$ is the input sample set, that is, the objects that have to be clustered, $c$ is the number of clusters, $\left\{c_{1}, c_{2}, \ldots, c_{n}\right\}$ the centroids of the clusters, that can be defined by a given matrix or randomly chosen, and $u_{i j}$ is the degree of membership of $x_{i}$ in the cluster $j$. Finally, the parameter $m$ is a real number greater than 1 that is a weighting factor called fuzzifier. Normally the Euclidean distance is used, but any norm $\left\|^{*}\right\|$ expressing the dissimilarity between any measured data and the center can be used.

Fuzzy partitioning is carried out through an iterative minimization of the objective function under the following fuzzy constraints:

In approach proposed by Bezdek (1981) in each iteration membership levels $u_{i j}$ and centroid positions $c_{j}$ are updated applying the technique of Lagrange multipliers. The algorithm stops when a maximum number of iterations is reached, or when the algorithm is unable to reduce the current value of the objective function.

Given that different organizational images can often be linked to an organization, in this work a soft clustering approach is considered more appropriate. Using FCM technique each organization is allowed to belong to many clusters with different degrees of membership and therefore have multiple images or metaphors linked. In the paper the results of the analysis are presented.

\section{Instrument for the identification of organizational images}

For the identification of images, an instrument developed by Johann (2004) was used. This instrument is a questionnaire with 35 questions on organizational aspects that are grouped into 7 blocks, each block is associated with one of the images considered. In order to identify characteristics of the images in an organization, a set of employees can make a quantitative assessment on each of the 35 questions of the questionnaire. The evaluation uses a discrete scale with values between 1 and 4 , according to the following criteria: 4 if there is strong presence, 3 if there is reasonable presence, 2 if there is little impact and 1 if there is virtually no presence.

The Annex shows the 35 questions selected and Table 2 shows the relationship of each question with one of the 7 images defined by Morgan.

\begin{tabular}{|l|c|c|c|c|c|c|c|} 
Image & $\mathbf{M}$ & $\mathbf{O}$ & $\mathbf{P S}$ & $\mathbf{B} / \mathbf{C}$ & $\mathbf{I D}$ & $\mathbf{F T}$ & $\mathbf{P P}$ \\
\cline { 2 - 6 }
\end{tabular}




\begin{tabular}{|c|c|c|c|c|c|c|c|}
\hline \multirow{4}{*}{ Question } & 1 & 2 & 3 & 4 & 5 & 6 & 7 \\
\cline { 2 - 8 } & 14 & 13 & 12 & 11 & 10 & 9 & 8 \\
\cline { 2 - 8 } & 15 & 16 & 17 & 18 & 19 & 20 & 21 \\
\cline { 2 - 8 } & 28 & 27 & 26 & 25 & 24 & 23 & 22 \\
\cline { 2 - 8 } & 29 & 30 & 31 & 32 & 33 & 34 & 35 \\
\hline
\end{tabular}

Table 1. Relationships between questions and organizational images.

With the answers to 35 questions, 7 numerical values can be generated with the sum of the scores for each of the 5 questions related to each of the 7 images. These 7 values can be taken into account in determining the most relevant image in the company, according to the opinion of the employee interviewed. An example of the tabulation of answers to the questionnaire is shown in Table 3. The sums of the scores associated with each of the images are shown in the last row, for example, in the case presented in Table 3, the organizational images most visible is "political system (PS)", but images M, C and ID also obtain high scores.

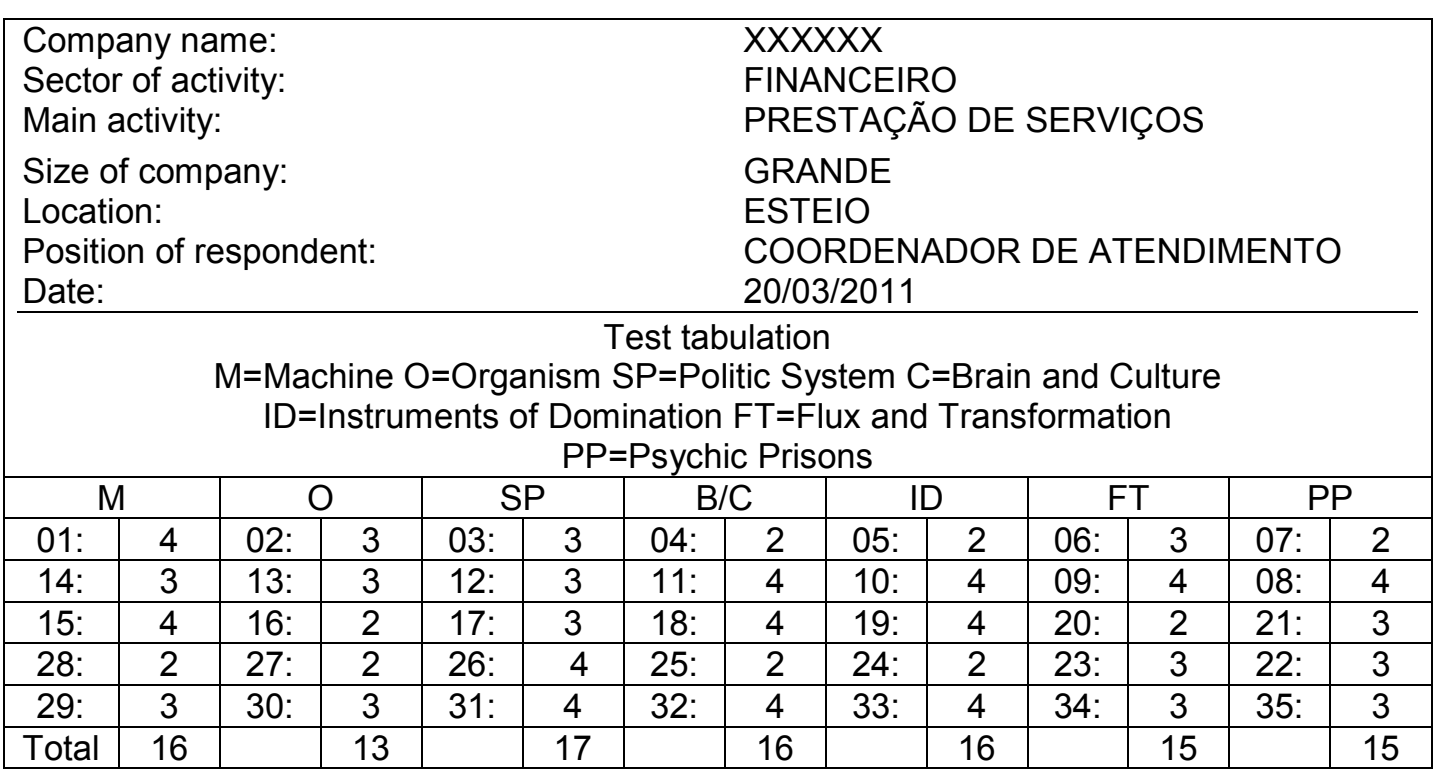

Table 2. Example of answers to the questionnaire.

\section{Case study}

To analyze the organizational images with greater presence in the state of Rio Grande do Sul (Brazil), a sample of 61 companies from various sectors and sizes was selected. In each company up to 4 employees were interviewed, yielding a total of 198 responses to the questionnaire (mean of 3.25 responses per company). All data were pre-processed for analysis with data mining techniques. In particular, clustering algorithms were applied to try to identify groups of companies responding, according to their employees, to similar images. We used a free software environment for statistical computing and graphics, this software is $\mathrm{R}$ and can be downloaded in the following site http://www.r-project.org/. This software implements a great variety of clustering algorithms; the Fuzzy C-Means (FCM) algorithm, implemented in package 'e1071', was selected.

The cmeans command needs several parameters to run: 
- The data matrix where columns correspond to variables and rows to observations. In our case 7 variables were considered with the average values corresponding to the sum of the scores of the 5 questions from each of the 7 blocks given by each employee in the company. The data matrix has 61 rows (companies).

- Number of clusters or initial values for cluster centers. In our case we decided to give 7 initial cluster centers. The center of cluster $i$ was initially defined as:

Note that 5 is the minimum value and 20 the maximum in a block of 5 questions.

- Maximum number of iterations, the value 500 was used.

- Distance measure to use; "euclidean" was used.

- Degree of fuzzification $(m) . m=2$ was used.

The algorithm needed a total number of 218 iterations to converge, and the final error was 3,8954. After the execution of the 218 iterations, the cluster centers were updated as is shown in Table 3 . Bold identifies the highest values in each centroid, that is, the images obtained higher scores in each group.

\begin{tabular}{|c|c|c|c|c|c|c|c|c|}
\hline & Image $M$ & Image O & $\begin{array}{c}\text { Image } \\
S P\end{array}$ & $\begin{array}{c}\text { Image } \\
B / C\end{array}$ & Image ID & $\begin{array}{c}\text { Image } \\
F T\end{array}$ & $\begin{array}{c}\text { Image } \\
P P\end{array}$ & $\begin{array}{c}\text { More } \\
\text { relevant } \\
\text { images } \\
\text { in the } \\
\text { cluster }\end{array}$ \\
\hline 1 & $\mathbf{1 4 . 0 6 9 6 9}$ & $\mathbf{1 4 . 1 4 3 4 0}$ & 10.75342 & 12.25663 & 12.81599 & $\mathbf{1 3 . 7 3 5 8 7}$ & 10.68910 & O, M, FT \\
\hline 2 & $\mathbf{1 3 . 4 3 6 2 1}$ & $\mathbf{1 4 . 9 1 0 5 5}$ & 11.87856 & 13.15049 & 12.30009 & $\mathbf{1 3 . 9 0 0 2 1}$ & 10.71262 & O, FT, M \\
\hline 3 & 13.87489 & 13.30586 & $\mathbf{1 4 . 3 2 8 9 9}$ & 12.72550 & $\mathbf{1 4 . 9 7 6 2 2}$ & $\mathbf{1 4 . 1 1 9 0 5}$ & 13.77686 & $\begin{array}{c}\text { ID, SP, } \\
\text { FT }\end{array}$ \\
\hline 4 & 14.89808 & $\mathbf{1 6 . 7 3 9 7 8}$ & 12.87971 & $\mathbf{1 5 . 2 8 3 7 9}$ & 14.23304 & $\mathbf{1 6 . 4 9 3 8 9}$ & 12.38194 & O, FT, C \\
\hline 5 & 14.21077 & $\mathbf{1 4 . 7 1 1 2 4}$ & 13.08771 & 13.61664 & $\mathbf{1 4 . 2 6 3 8 2}$ & $\mathbf{1 4 . 5 2 7 3 6}$ & 12.50887 & $\begin{array}{c}\text { O, FT, } \\
\text { ID }\end{array}$ \\
\hline 6 & $\mathbf{1 5 . 0 3 5 8 5}$ & $\mathbf{1 5 . 9 8 6 6 9}$ & 12.03754 & 14.72027 & 13.31240 & $\mathbf{1 4 . 9 5 1 3 8}$ & 11.05963 & O, M, FT \\
\hline 7 & 14.31467 & $\mathbf{1 4 . 6 0 6 6 8}$ & 13.31190 & 13.63852 & $\mathbf{1 4 . 3 9 3 3 6}$ & $\mathbf{1 4 . 5 6 1 4 3}$ & 12.62568 & $\begin{array}{c}\text { O, FT, } \\
\text { ID }\end{array}$ \\
\hline
\end{tabular}

Table 3. Cluster centers and more relevant images after the execution of FCM algorithm.

As a result of the algorithm we obtained a matrix with the degrees of membership of each company for each of the 7 groups identified. Table 4 shows this matrix, the membership levels can also be shown using a density plot, as in Figure 1. For example, company 1 has the following levels of group membership:

In continuation is the ranking of the images presented in each group as well as the graphic clustering.

\begin{tabular}{|l|l|l|l|l|l|l|l|}
\hline Cluster & Image 1 & Image 2 & Image 3 & Image 4 & Image 5 & Image 6 & Image 7 \\
\hline 1 & $\mathrm{O}$ & $\mathrm{M}$ & $\mathrm{FT}$ & $\mathrm{ID}$ & $\mathrm{B} / \mathrm{C}$ & $\mathrm{SP}$ & $\mathrm{PP}$ \\
\hline 2 & $\mathrm{O}$ & $\mathrm{FT}$ & $\mathrm{M}$ & $\mathrm{C}$ & $\mathrm{ID}$ & $\mathrm{SP}$ & $\mathrm{PP}$ \\
\hline 3 & $\mathrm{ID}$ & $\mathrm{SP}$ & $\mathrm{FT}$ & $\mathrm{M}$ & $\mathrm{PP}$ & $\mathrm{O}$ & $\mathrm{C}$ \\
\hline
\end{tabular}




\begin{tabular}{|l|l|l|l|l|l|l|l|}
\hline 4 & $\mathrm{O}$ & $\mathrm{FT}$ & $\mathrm{C}$ & $\mathrm{M}$ & ID & SP & PP \\
\hline 5 & $\mathrm{O}$ & FT & ID & M & C & SP & PP \\
\hline 6 & $\mathrm{O}$ & $\mathrm{M}$ & FT & C & ID & SP & PP \\
\hline 7 & $\mathrm{O}$ & FT & ID & M & C & SP & PP \\
\hline
\end{tabular}

Table 4. Ranking of the images
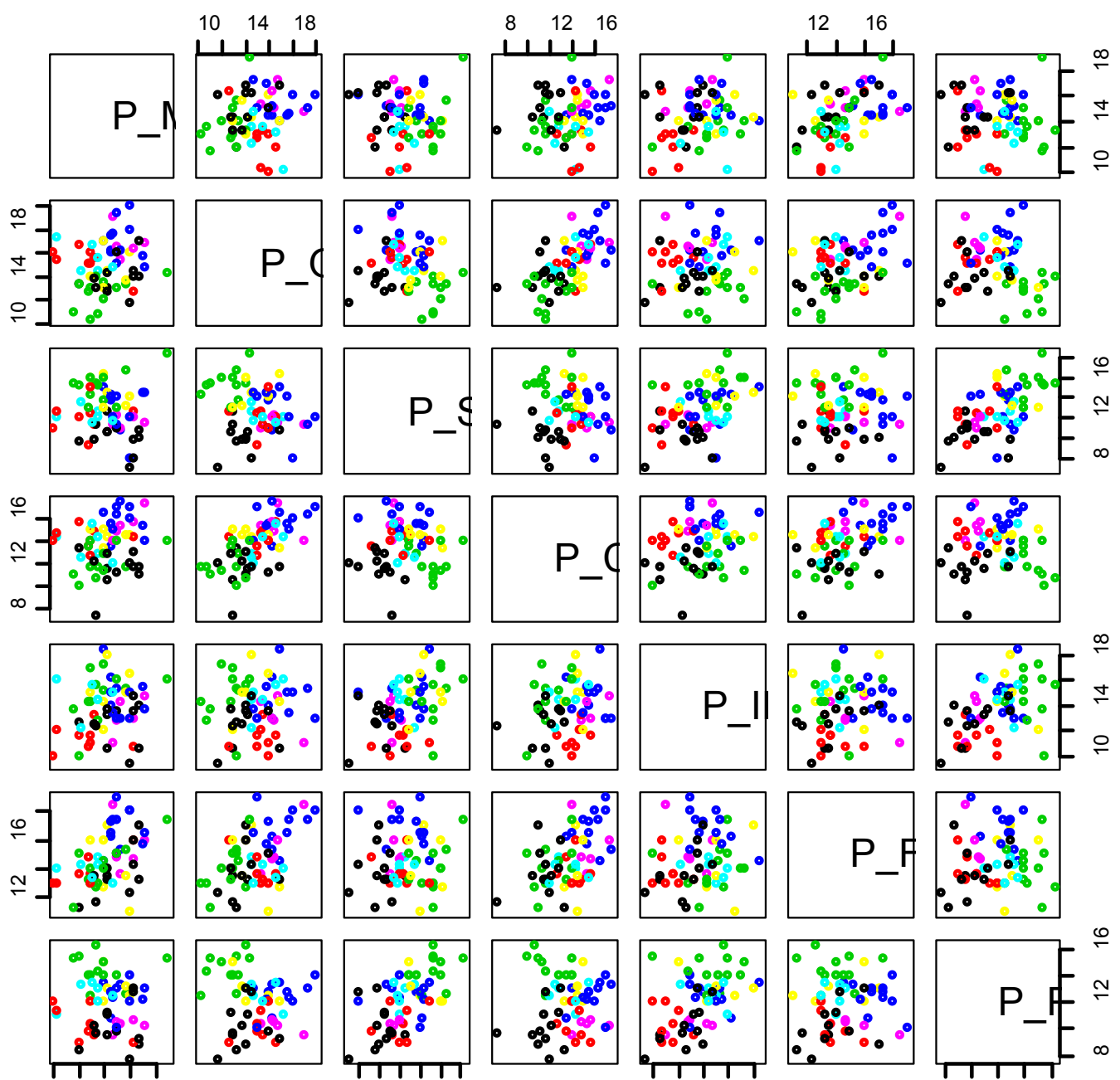

$10 \quad 14 \quad 18$
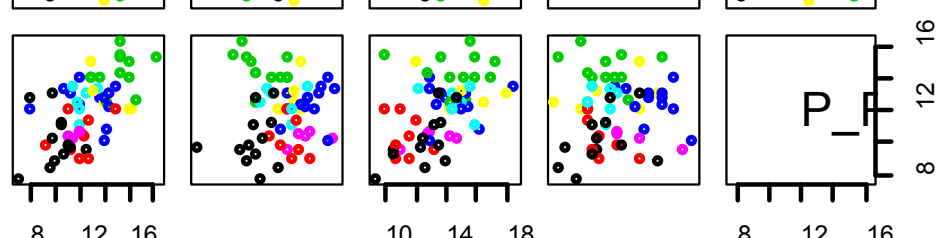

Figure 1: graphic clustering

According to these values, company 1 would be more clearly associated with clusters 4,6 and 7, groups characterized by a high presence of $O$ and FT images.

\begin{tabular}{|c|c|c|c|c|c|c|c|}
\hline & Cluster 1 & Cluster 2 & Cluster 3 & Cluster 4 & Cluster 5 & Cluster 6 & Cluster 7 \\
\hline 1 & 0.07066084 & 0.08658671 & 0.10323928 & 0.21063065 & 0.18665701 & 0.15041535 & 0.19181016 \\
\hline 2 & 0.07312988 & 0.08607538 & 0.15161303 & 0.20526897 & 0.17179575 & 0.13193341 & 0.18018358 \\
\hline 3 & 0.06859525 & 0.07444648 & 0.19060567 & 0.17475744 & 0.18399386 & 0.10722599 & 0.20037532 \\
\hline 4 & 0.03169135 & 0.04403128 & 0.04741090 & 0.57385733 & 0.09821968 & 0.10476523 & 0.10002422 \\
\hline 5 & 0.08308866 & 0.09396503 & 0.06310459 & 0.26138938 & 0.11674858 & 0.26730794 & 0.11439583 \\
\hline 6 & 0.12172084 & 0.15924327 & 0.10787176 & 0.13952651 & 0.16655198 & 0.14846257 & 0.15662307 \\
\hline 7 & 0.12279663 & 0.15318111 & 0.06888526 & 0.20865810 & 0.11651387 & 0.21916242 & 0.11080261 \\
\hline 8 & 0.04220773 & 0.07790881 & 0.01870347 & 0.07060410 & 0.05522701 & 0.68612636 & 0.04922252 \\
\hline 9 & 0.08219092 & 0.10195635 & 0.06719794 & 0.25448306 & 0.13011700 & 0.23951137 & 0.12454337 \\
\hline
\end{tabular}




\begin{tabular}{|c|c|c|c|c|c|c|c|}
\hline 10 & 0.05546326 & 0.06238707 & 0.11696803 & 0.04217665 & 0.36042338 & 0.05174491 & 3671 \\
\hline 11 & 0.28728923 & 0.24772864 & 0.08701666 & 0.05989234 & 0.11032077 & 0.10274618 & 0.10500617 \\
\hline 12 & 0.22593052 & 0.15658323 & 0.08749638 & 0.11131703 & 0.13027708 & 0.16301567 & 0.12538010 \\
\hline 13 & 0.06920147 & 0.07903842 & 0.12510591 & 0.19929293 & 0.18164698 & 0.14795461 & 0.19775967 \\
\hline 14 & 0.08285978 & 0.11859962 & 0.15931363 & 0.12035732 & 0.19264877 & 0.12761317 & 60771 \\
\hline 15 & 0.12177958 & 0.13361607 & 0.04982584 & 0.10184959 & 0.12635401 & 0.35147288 & 0.11510203 \\
\hline 16 & 0.14045734 & 0.26530835 & 0.09467698 & 0.08950505 & 0.14555268 & 0.13023758 & 0.13426202 \\
\hline 17 & 0.08681304 & 0.09328667 & 0.19122849 & 0.16892750 & 0.16149141 & 0.12522848 & 0.17302441 \\
\hline 18 & 0.07497735 & 0.08073145 & 0.30462401 & 0.09105763 & 0.17141417 & 0.08550092 & 0.19169448 \\
\hline 19 & 0.08341590 & 0.08578571 & 0.35908959 & 0.07812405 & 0.15503223 & 0.07478851 & 0.16376401 \\
\hline 20 & 0.17479429 & 0.30830196 & 0.04631832 & 0.07003819 & 0.10548488 & 0.20155637 & 0.09350599 \\
\hline 21 & 0.09634673 & 0.10469832 & 0.15798910 & 0.11641307 & 0.18966021 & 0.13881451 & 0.196 \\
\hline 22 & 0.17231070 & 0.18867734 & 0.08467363 & 7158 & 0.13 & 0.18768695 & \\
\hline 23 & 0.13553735 & 0.65585995 & 0.02651960 & 5840 & 1346 & 0.05677979 & 0.04703145 \\
\hline 24 & 0.26976889 & 0.29072870 & 0.05170888 & 0.07291884 & 0.09093024 & 0.14135547 & 0.08258898 \\
\hline 25 & 0.13949925 & 0.26258352 & 0.06216253 & 0.11056620 & 0.11528520 & 0.20376653 & 0.10613677 \\
\hline 26 & 0.12697591 & 0.11798789 & 0.30413710 & 37029 & 3258 & 50053 & 29570 \\
\hline 27 & 0.06342657 & 0.08722344 & 0.14 & 0639 & 697 & 9781 & 2616 \\
\hline 28 & 0.06385745 & 0340 & 0.0 & 1793 & 2439 & 3607 & 1943 \\
\hline 29 & 0.07901129 & 1119 & 0.0 & 361 & 1321 & 4222 & 848 \\
\hline 30 & 0.05739890 & 0.06748329 & 0.10023415 & 3224 & 0.16538393 & 0.16067014 & 0.17949735 \\
\hline 31 & 0.28326301 & 0.20365793 & 0.05764492 & 54032 & 0.11426179 & 0.15872703 & 0.10390500 \\
\hline 32 & 0.28461438 & 0.24248080 & 0.07755410 & 8642 & 1574 & 7521 & 7336 \\
\hline 33 & 0.06903735 & 823 & 0.0 & 0.3 & 726 & 823 & 3056 \\
\hline 34 & 0.01807095 & 6151 & 1633 & 6586 & 6922 & 0.018 & 4898 \\
\hline 35 & 0.11663941 & 1074 & 997 & 4561 & 5682 & 5965 & 4780 \\
\hline 36 & 0.07817857 & 0.08536278 & 0.34888223 & 9400 & 3021 & 0.06024328 & 0.19080894 \\
\hline 37 & 0.10714989 & 0.11048183 & 0.32739166 & 067 & 308 & 7875 & 0.15927413 \\
\hline 38 & 0.69337887 & 84761 & 9171 & 3147 & 3369 & 23923 & 0.04557743 \\
\hline 39 & 0.17273715 & 0.2 & & 023 & 008 & 0.13496245 & 2493 \\
\hline 40 & 0.03241751 & 640 & 0.0 & 1622 & 600 & 0.10528039 & 0.09668645 \\
\hline 41 & 0.22437489 & 3734 & 0018 & 0541 & 7166 & 0.18305758 & 0.10430293 \\
\hline 42 & 0.10765563 & 0.21007287 & 6404 & 4859 & 0240 & 0.14315675 & 0.16009971 \\
\hline 43 & 0.11133460 & 0.18090745 & 0.16155856 & 0.06774603 & 0.20270129 & 0.08630506 & 0.18944702 \\
\hline 44 & 0.13048799 & 0.14819301 & 0.20418735 & 0.09798384 & 0.15786594 & 0.10286958 & 0.15841229 \\
\hline 45 & 0.05764661 & 4164 & 0.2 & 307 & 059 & 0928 & 3545 \\
\hline 46 & 0.25750525 & 599 & & 01 & & 0.1405 & \\
\hline 47 & 0.04572654 & 0.06161214 & 0.03807164 & 0.52587451 & 0.08298042 & 0.16708356 & 5120 \\
\hline 48 & 0.05939674 & 0.06714226 & 0.38750100 & 0.08041471 & 0.15888866 & 0.06972908 & 0.17692754 \\
\hline 49 & 0.08586828 & 0.11065564 & 0.08367132 & 0.26059800 & 0.13202895 & 0.19531702 & 0.13186079 \\
\hline 50 & 0.16231335 & 0.11824507 & 0.13174813 & 0.05896488 & 0.22471806 & 0.09544003 & 0.20857048 \\
\hline 51 & 0.06117608 & 0.06424937 & 0.17273495 & 0.07234683 & 0.25039101 & 0.09209677 & 0.28700499 \\
\hline 52 & 0.09123197 & 0.11071931 & 0.16669303 & 0.12941725 & 0.18977795 & 0.11894709 & 0.19321339 \\
\hline 53 & 0.59796974 & 0.18279490 & 0.032 & 6130 & 0.05 & 0.05685057 & 0.04861317 \\
\hline 54 & 0.43332432 & 0.15658670 & 0.06662395 & 0.04240830 & 0.11423622 & 0.08300395 & 0.10381654 \\
\hline 55 & 0.11348891 & 0.11319047 & 0.07704351 & 0.24432758 & 0.12596022 & 0.20477560 & 0.12121372 \\
\hline 56 & 0.26191311 & 0.19090609 & 0.12260103 & 0.07201902 & 0.12645872 & 0.10397421 & 0.12212780 \\
\hline 57 & 0.17894663 & 0.12674166 & 0.09227940 & 0.12114093 & 0.16112533 & 0.16691343 & 0.15285262 \\
\hline 58 & 0.07228857 & 0.11036817 & 0.05615977 & 0.08543059 & 0.29143982 & 0.16136069 & 0.22295239 \\
\hline 59 & 0.08730678 & 0.10696052 & 0.10468709 & 0.16331970 & 0.19488071 & 0.15817972 & 0.18466549 \\
\hline 60 & 0.07500784 & 0.07422026 & 73367 & 0.07 & 5454 & 0.07041335 & 0.15878586 \\
\hline 61 & 0.23432493 & 0.13894778 & 0.09988333 & 0.10115031 & 0.14476995 & 0.14358131 & 0.13734238 \\
\hline
\end{tabular}

Table 5. Membership levels in the fuzzy clustering process. 


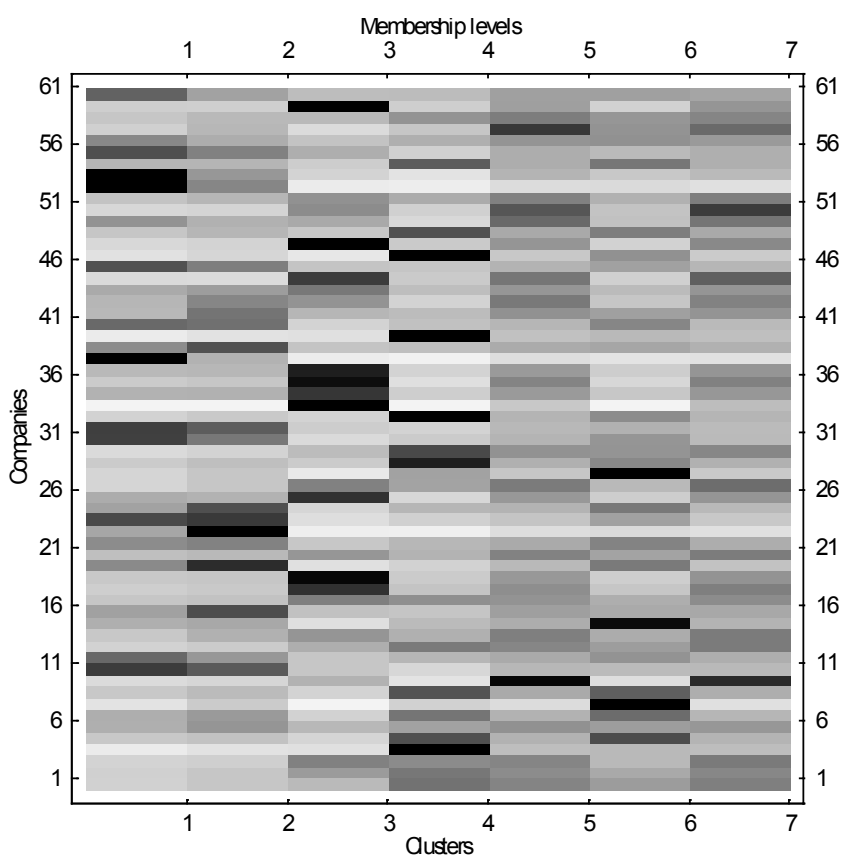

Figure 2. Density plot of membership levels in the fuzzy clustering process.

The $\mathrm{R}$ cmeans command also generates the closest hard clustering solution. This information is also useful for identifying significant groups. Table 6 shows the number of companies in the 7 hard clusters after the execution of the algorithm. 3 groups are bigger than the rest, clusters 1, 3 and 4 .

\begin{tabular}{|c|c|}
\hline Cluster & Number of companies \\
\hline 1 & 12 \\
\hline 2 & 8 \\
\hline 3 & 12 \\
\hline 4 & 12 \\
\hline 5 & 6 \\
\hline 6 & 5 \\
\hline 7 & 6 \\
\hline
\end{tabular}

Table 6. Cluster sizes in the closest hard clustering.

\section{Conclusions}

The results of applying the techniques of soft clustering confirm the difficulty of associating a single image or metaphor to a company, as features of the other images are also present. However, according to these results, it is noted that the most visible image in the organizations studied is "organisms". This image is the most relevant in 6 out of the 7 groups identified. This metaphor means seeing the businesses as behaving in similar ways to our own biological mechanisms, successful businesses are often adaptable and open to change and the structures and procedures are less fixed.

Also remarkable is that the image "flux and transformation" appears in all groups with a high value. So characteristics as constant change, dynamic equilibrium, flow, self-organization, systemic wisdom, attractors, chaos, complexity, butterfly effect, emergent properties, dialectics, paradox are also present in most of companies.

All groups obtained seem to have a very similar structure, with similar most relevant images, but one of them (cluster 3 ) is clearly different from the 
rest, with a high degree of association with images that are less present in the other groups, such as images "political system" and "instrument of domination".

In summary, this work confirms the difficulty of linking a company with a single image, but it has allowed seeing images that have a greater presence in companies operating in Rio Grande do Sul. The sample used is small; it would be interesting to expand its size by analyzing other companies or even carrying out sectorial or geographical studies.

\section{References}

BEZDEK, J. C. Pattern recognition with fuzzy objective function algorithms. Plenum Press, New York: 1981.

DUNN, J. C. A fuzzy relative of the ISODATA process and its use in detecting compact well-separated clusters, Journal of Cybernetics 3, pages 32-57. 1973.

HAN, J.; KAMBER, M. Data Mining. Concepts and Techniques (2 ${ }^{\text {nd }}$ Edition). Morgan Kaufmann Publishers: 2006.

JOHANN, S. L. Gestão da cultura corporativa - como as organizações de alto desempenho gerenciam a sua cultura organizacional. São Paulo: Editora Saraiva. 2004.

KRUSE, R.; HOPPNER, F.; KLAWONN, F.; RUNKLER, T. Fuzzy Cluster Analysis. John Wiley and Sons: 1999.

MORGAN, G. Images of Organization (1st ed.). SAGE Publications, Newbury Park, CA. 1986.

WITTEN, I.; FRANK, E. Data Mining. Practical Machine Learning Tools and Techniques. Morgan Kaufmann Publishers. 2005.

ZADEH, L. Fuzzy Sets. Information and Control, 8, pp. 338-352. 1965.

\section{Annex: Questionnarie for the identification of organizational images}

1) Procedures, operations and processes are standardized.

2) Changes in the organization are normally a reaction to changes that already occurred in the macro business environment.

3) Administrators frequently talk about authority, power and superior-subordinate relationships.

4) Flexible and creative action.

5) Working in inadequate circumstances and conditions is considered a proof of loyalty to the organization.

6) The organization sees itself as a part of a larger system where there is an interdependence that involves the community, suppliers and the competition.

7) People and groups tend to display infantile behavior.

8) Past achievements are constantly cited as references and as examples on how to deal with present situations and how to face future adversities.

9) The organization evolves in harmony and balance with its macro environment.

10) People act under constant stress and pressure.

11) There is constant questioning and redirection of actions.

12) Power serves to provide discipline and achieve order in conflicts of interest.

13) The organization considers the motivations and needs of people. 
14) There are rigid patterns and uniformity in people's behavior.

15) The company has and utilizes a great number of rules, norms and regulations about operational aspects of the business.

17) The delegation of power to operational levels tends to be very restricted.

18) Negative feedback is encouraged to correct the organizational direction.

19) The organization expects complete devotion and dedication from its employees.

20) The company benefits more from external events (environmental, etc.) than from strict planning.

21) There are many taboos and prejudices in the organization.

22) The relationships between superiors and subordinates tend to contain elements of love and hate.

23) Long term achievements will be achieved in partnership with the forces acting with the macro-environment and not against it.

24) To dismiss people and streamline activities are part of the game.

25) Most people think about and influence on the destiny of the company.

26) Interpersonal gossip consumes energy and diverts attention from productivity.

27) Organizational objectives and people's needs can be met simultaneously.

28) The organization is a realm of bureaucracy.

29) The organization is expected to operate in a routine, efficient, reliable and predictable manner.

30) Employees are seen as valuable resources who can offer rich and varied contributions to the organizations activities, provided that the organization attends to their needs and motivations.

31) Rumors and gossip are frequent.

32) The organization tends to offer quick answers to changes in their macro-environment.

33) The organization values executives who appear framed and faithful to the mode of being of the company

34) In strategic decision making the company normally abandons the simple view and prefers to take into account the complexity of the situation.

35) People are dedicated to the organization because they feel they belong to something greater, which transcends their existence and individual limitations. 